\title{
Primary Extragastrointestinal Stromal Tumor of Retroperitoneum: Poor Response to Tyrosine Kinase Inhibitor
}

\section{Min-Young Lee}

Division of Hematology and Oncology, Department of Internal Medicine, Soonchunhyang University Seoul Hospital, Soonchunhyang University College of Medicine, Seoul, Korea

\begin{abstract}
Gastrointestinal stromal tumors (GIST) are the most common sarcomas arising in the gastrointestinal tract. Stromal tumors arising outside the gastrointestinal tract are known as extragastrointestinal stromal tumors (EGIST). EGISTs may occur in omentum, mesentery, or retroperitoneum and are histologically and immunophenotypically similar to that in GISTs. EGISTs are very rare, so pathogenesis, incidence, and prognosis of EGISTs are not defined yet. In particular, there are a few reported literatures on the treatment of metastatic EGISTs. I report a case of a primary EGIST of the retroperitoneum with lung metastasis surgically removed and treated with tyrosine kinase inhibitor.
\end{abstract}

Keywords: Gastrointestinal stromal tumors; Retroperitoneum; Protein-tyrosine kinases

\section{INTRODUCTION}

Gastrointestinal stromal tumor (GIST) is the most common sarcomas arising in the gastrointestinal tract [1]. GISTs are more frequently found in stomach (50\%), followed by small intestine (25\%), colon/rectum (5\%-10\%), and esophagus (5\%). GIST that arises primarily outside the gastrointestinal tract is known as extragastrointestinal stromal tumor (EGIST). Approximately $80 \%$ of EGISTs are located in the omentum or mesentery, and the rest develops in the retroperitoneum [2]. EGIST shows a c-kit (CD117) expression and histologic appearance similar to those of GIST.

About $80 \%$ of GISTs have a mutation in the gene encoding the KIT receptor tyrosine kinase; another $5 \%$ to $10 \%$ of GISTs have a mutation in the gene encoding the related PDGFRA receptor tyrosine kinase [3,4]. Metastatic GISTs have previously been documented to be resistant to conventional chemotherapies. Since KIT or PDGFRA mutation occurs in the majority of cases of GIST, tyrosine kinase inhibition has developed as the primary therapy along with surgery for the treatment of GIST. The US Food and Drug Administration now approved tyrosine kinase inhibitors
(TKIs), such as imatinib, sunitinib, and regorafenib for the treatment of metastatic GIST. Recently, some reports have demonstrated that the pattern of KIT and PDGFRA mutation in EGIST was also similar to that in GIST. However, the treatment of metastatic EGIST is not established because of rarity of the disease. I report a case of metastatic EGIST surgically removed and treated with sequential multiple TKI.

\section{CASE REPORT}

A 55-year-old man was referred to Soonchunhyang University Seoul Hospital for mass of retroperitoneum. He presented abdominal discomfort and flank pain 1 month ago. Before visiting our hospital, he performed abdominal ultrasonography and revealed abdominal mass. He had no significant past medical or surgical history except of gastro-esophageal reflux disease. In our hospital, he underwent abdominal computed tomography (CT) scan that showed a retroperitoneal heterogeneous enhancing mass $(9.0 \times$ $8.5 \times 8.1 \mathrm{~cm}$ ) in left iliopsoas muscle (Fig. 1). The patient performed ultrasonography-guided gun biopsy with the result of prolifera-
Correspondence to: Min-Young Lee

Division of Hematology and Oncology, Department of Internal Medicine, Soonchunhyang University Seoul Hospital, Soonchunhyang University College of Medicine, 59 Daesagwan-ro, Yongsan-gu, Seoul 04401, Korea

Tel: +82-2-710-3278, Fax: +82-2-710-3194, E-mail: redmin07@gmail.com

Received: May 31, 2018 / Accepted after revision: Jun. 11, 2018
(C) 2018 Soonchunhyang Medical Research Institute This is an Open Access article distributed under the terms of the Creative Commons Attribution Non-Commercial License (http://creativecommons.org/licenses/by-nc/4.0/). 


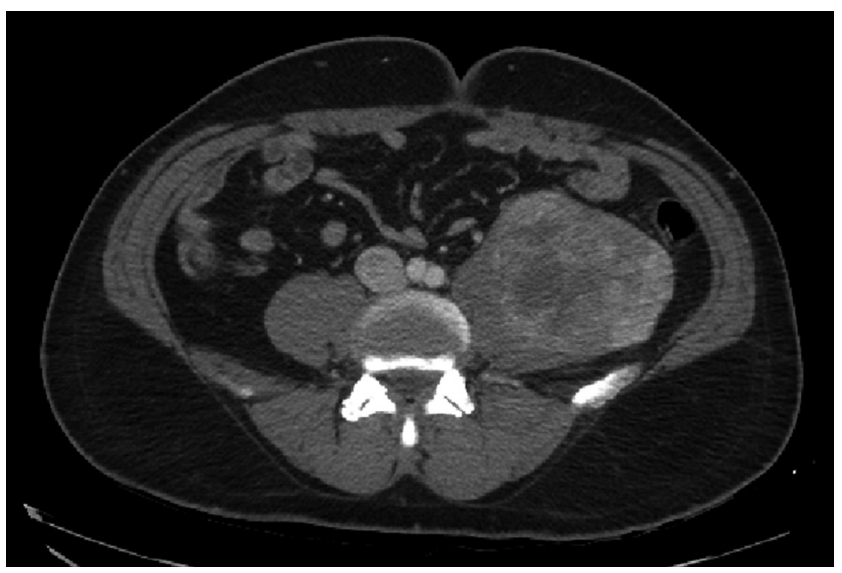

Fig. 1. Abdominal computed tomography scan shows about $9.0 \times 8.5 \times 8.1-\mathrm{cm}$ sized heterogeneous enhancing mass in left psoas muscle.

tion of atypical spindle cells, positive immunohistochemical staining for CD117. To know his disease status, the patient underwent routine blood test, electrocardiogram, gastroduodenoscopy, and colonoscopy, and the results were unremarkable. However, CT scan of chest showed about $1.2-\mathrm{cm}$ sized enhancing discrete nodule in left superior lingular division, suggestive of hematogenous metastasis (Fig. 2). A multidisciplinary team of orthopedic, thoracic surgeons and medical oncologist discussed about resectability of retroperitoneal and lung mass. We decided palliative surgery for his retroperitoneal and lung mass because he had potentially resectable disease.

The patient underwent a complete gross excision of the retroperitoneal mass and wedge resection of left upper lung lingluar segment via video-assisted thoracoscopic surgery (VATS). Histologically, the excisional retroperitoneal and lung mass tumor cells consisted of proliferation of spindle cells. Average mitotic count was $>10$ per 50 high power filed (HPF) and Ki-67 labeling index $>20 \%$. Immunohistochemical analysis of retroperitoneal mass showed diffusely strong CD117 positivity, focal positivity for CD34, actin, and CD 56, while it was negativity for desmin and S-100. Also, lung nodule showed positivity for CD117, actin, and vimentin and negativity for CD34, S-100, and CK. The microscopy results indicated that the mass from the retroperitoneum was spindle cell type EGIST and the mass from the lung was metastatic EGIST. A molecular genetic analysis for the KIT gene (exons 9, 11, 13 , and 17) and the PDGFRA gene (exons 12, 14, and 18) mutation was performed by direct sequencing of polymerase chain reaction products. His tumor had no mutation of KIT and PDGFRA genes.

Postoperatively, the patient started treatment with imatinib me-

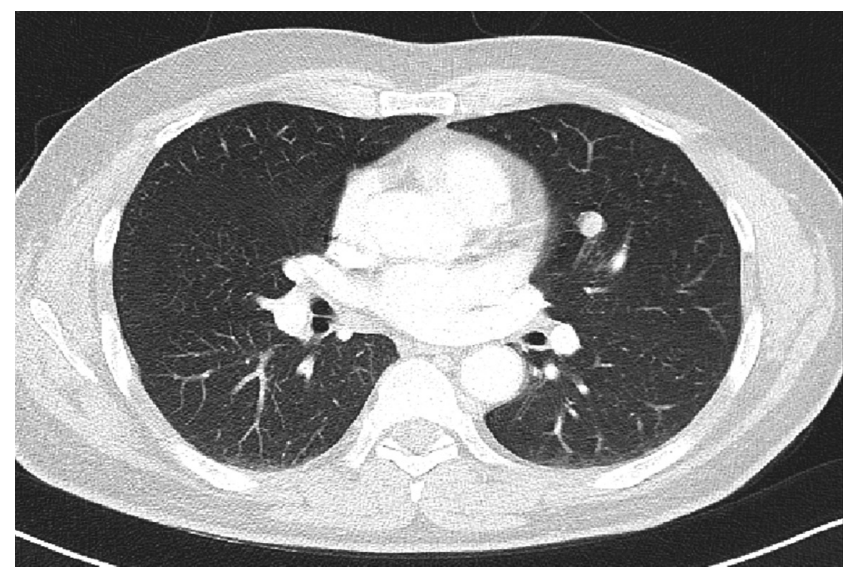

Fig. 2. Chest computed tomography scan shows about 1.2-cm sized enhancing discrete nodule in left superior lingular division.

sylate within 3 weeks. First starting dose was $400 \mathrm{mg}$ once daily, but dose reduction was done because of hepatotoxicity. His laboratory test showed severe hepatic impairment that total bilirubin was greater than 3 times the upper limit of normal (ULN) and SGOT/SGPT (serum glutamate oxaloacetate transaminase/serum glutamate pyruvate transaminase) were greater than 3 to 5 times the ULN. Reduction dose was $300 \mathrm{mg}$ once daily. After 5 months, CT scan of chest showed about 1.7 -cm sized soft tissue density lesion at almost resection site of the left lingular division. Abdominal CT scan was revealed no definitive evidence of local tumor recurrence in left iliopsoas muscle. Our multidisciplinary team discussed about resectability of the lung and decided metastatectomy of the lung. The patient underwent segmentectomy of anterior and superior lingular segment of left upper lung via VATS. In advanced and after progression to imatinib, the patient started treatment with sunitinib malate. The treatment schedule was started standard schedule of 4-weeks-on and 2-weeks-off treatment (4/2 schedule). However, he temporary interrupted of therapy with sunitinib because tonsillectomy due to severe infectious tonsillitis. After that surgery, the patient started alternative schedule, such as sunitinib 50 mg per day, 2-weeks-on and 1-week-off treatment (2/1 schedule). Sunitinib treatment was maintained for 10 months without any specific toxicity. During follow-up, the patient began to complain of anterior chest wall pain. The abdominal and chest CT scan showed recurrent disease of left iliopsoas muscle and multiple metastatic lesions including lung, lymph node, and bone of low sternal body, left second rib, and left iliac. Despite treatment with sunitinib, his disease had become unresectable metastatic disease state. The patient changed treatment with regorafenib and 
maintained for 3 months. In the following response evaluation, the disease showed further progress. The abdominal and chest CT scan showed newly appeared multiple nodules of lung and interval increased size of multiple lymph nodes and enhancing mass at left psoas muscles. Also, there was more increased in extent of bone destruction at the left second rib, sternal body with soft tissue mass, and left iliac bone. The soft tissue mass in sternal body extended to the pericardium and showed mild compression to the anterior aspect of heart. Currently, he wanted stop more treatment but physician recommended cytotoxic chemotherapy.

\section{DISCUSSION}

GIST is the most common mesenchymal tumor of the gastrointestinal tract [1]. Approximately $80 \%$ of GISTs are caused by gainof function mutation in the proto-oncogene KIT, which encodes a tyrosine kinase receptor. Roughly $8 \%$ of GISTs are associated with gain-of-function mutations in the structurally similar tyrosine kinase receptor gene PDGFRA, encoding the platelet-derived growth factor receptor $[3,4]$. Understanding the molecular pathophysiology of this condition has allowed rational development of agents that target signaling aberrations in the cancer cells. Therefore, GIST represents one of the paradigms of targeted agent era. Advances in molecular targeted therapy have led to the treatment of TKI for GISTs, including imatinib, sunitinib, and regorafenib $[5,6]$.

EGIST is defined as soft tissue sarcomas derived from an external organs of the gastrointestinal tract that is histologically similar to GIST and positive for immunostaining of CD117 by Reith et al. [7]. EGISTs are extremely rare neoplasm, which account for less than $5 \%$ in large series of stromal tumors. Although EGISTs seem to have morphological and immunohistochemical similarities with GISTs, their pathogenesis, incidence, and prognosis are not completely known because they are very rare [8]. Recently, Yamamoto et al. [2] reported mutational status of EGISTs, that the pattern of KIT and PDGFRA mutations in EGISTs were similar to that in GIST. They suggest that the KIT and PDGFRA mutations play an important role in the tumorigenesis of EGIST. It is clinically important that EGIST have c-kit (CD117) expression and KIT mutations because KIT is an important molecule, not only for the pathologic diagnosis but also as a therapeutic target.

The standard treatment of both GISTs and EGISTs is a complete resection when the tumors show having resectability or no metastasis to other organs. However, the treatment for metastatic
EGISTs has not yet been established. To date, chemotherapy with multiple TKIs for GISTs is generally used to treat advanced EGISTs. Some literatures have reported curative cases of EGISTs using imatinib in preoperative or postoperative $[9,10]$. There is no report of treatment with TKIs other than imatinib in metastatic advanced EGISTs.

The current report presented a rare case of metastatic and recurrent EGIST of retroperitoneum that was treated surgical resection and chemotherapy with multiple TKIs, including imatinib, sunitinib and regorafenib. Approximately $10 \%$ of patients with a GIST have primary resistance to responses to imatinib, defined as progression within the first 6 months of treatment. Clinical responses to imatinib correlate with tumor genotype, and many resistant tumors lack mutations in KIT or PDGFRA, or they harbor a PDGFRA D842 mutation. Most of these tumors will lack KIT expression by immunohistochemistry. In this case, the patient had resistance to imatinib even though there were no KIT and PDGFRA mutations. It is difficult to explain the genetic causes for the treatment resistance so far. In another aspect, Yamamoto et al. [2] reviewed 39 cases of EGISTs and concluded that tumors with high mitotic counts ( $\geq 5 / 50 \mathrm{HPF}$ ), or a high ki-67 labeling index ( $\geq$ $10 \%)$, were significantly correlated with worse prognosis. In our case, the tumor had high risk factors with large size $(9.0 \mathrm{~cm}$ in greatest dimension), high mitotic rate (>10/50 HPF) and high Ki67 labeling index (>20\%). The patient underwent treatment with proven TKI for treatment of GIST but showed progression and recurrence of disease. Further analyses with a large number of metastatic EGISTs are needed to determine efficacy and safety of chemotherapy with selective TKIs.

\section{REFERENCES}

1. Ducimetiere F, Lurkin A, Ranchere-Vince D, Decouvelaere AV, Peoc'h M, Istier L, et al. Incidence of sarcoma histotypes and molecular subtypes in a prospective epidemiological study with central pathology review and molecular testing. PLoS One 2011;6:e20294.

2. Yamamoto H, Oda Y, Kawaguchi K, Nakamura N, Takahira T, Tamiya S, et al. c-kit and PDGFRA mutations in extragastrointestinal stromal tumor (gastrointestinal stromal tumor of the soft tissue). Am J Surg Pathol 2004;28:479-88.

3. Fletcher CD, Berman JJ, Corless C, Gorstein F, Lasota J, Longley BJ, et al. Diagnosis of gastrointestinal stromal tumors: a consensus approach. Int J Surg Pathol 2002;10:81-9.

4. Heinrich MC, Corless CL, Duensing A, McGreevey L, Chen CJ, Joseph N, et al. PDGFRA activating mutations in gastrointestinal stromal tumors. Science 2003;299:708-10.

5. Demetri GD, Reichardt P, Kang YK, Blay JY, Rutkowski P, Gelderblom H, 
et al. Efficacy and safety of regorafenib for advanced gastrointestinal stromal tumours after failure of imatinib and sunitinib (GRID): an international, multicentre, randomised, placebo-controlled, phase 3 trial. Lancet 2013;381:295-302.

6. Demetri GD, van Oosterom AT, Garrett CR, Blackstein ME, Shah MH, Verweij J, et al. Efficacy and safety of sunitinib in patients with advanced gastrointestinal stromal tumour after failure of imatinib: a randomised controlled trial. Lancet 2006;368:1329-38.

7. Reith JD, Goldblum JR, Lyles RH, Weiss SW. Extragastrointestinal (soft tissue) stromal tumors: an analysis of 48 cases with emphasis on histo- logic predictors of outcome. Mod Pathol 2000;13:577-85.

8. Trombatore C, Palmucci S, Angelico G, Vasquez E, Petrillo G, Puleo S, et al. Extragastrointestinal stromal tumor of lesser omentum: a challenging radiological and histological diagnosis. Clin Imaging 2015;39:1123-7.

9. Muto M, Fujiya M, Okada T, Inoue M, Yabuki H, Kohgo Y. An invasive extragastrointestinal stromal tumor curably resected following imatinib treatment. J Gastrointestin Liver Dis 2013;22:329-32.

10. Malhotra A, Wright J, Gajra A. Extra gastrointestinal stromal tumor treated with imatinib in a patient with Neurofibromatosis type 1. J Gastrointest Oncol 2012;3:373-6. 